\title{
Prognostic significance of microvascular invasion in tumor stage for hepatocellular carcinoma
}

\author{
Yong Keun Park ${ }^{1,2}$, Sung Kyu Song ${ }^{2}$, Bong-Wan Kim', Seung-Keun Park³, Chul-Woon Chung ${ }^{2}$ \\ and Hee-Jung Wang ${ }^{1 *}$
}

\begin{abstract}
Background: The presence of microvascular invasion (McVI) in hepatocellular carcinoma (HCC) has been proposed as a cause of recurrence and poor survival, although this has not been officially emphasized in staging systems. Thus, we conducted a retrospective study to investigate the prognostic importance of McVl in tumor staging in patients with HCC who underwent hepatic resection.

Methods: A retrospective analysis was performed of patients who underwent hepatic resection for HCC at our center from 1994 to 2012. Patients with HCC were classified into four groups based on the presence of McVI and extent of gross vascular invasion (VI).

Results: The 5-year overall and recurrence-free survival rates of 676 patients were 63.3 and $42.6 \%$, respectively. There was no difference in tumor recurrence or survival rate between patients with HCC and McVI without gross VI and those with gross VI confined to segmental/sectional branches. Multivariate analysis revealed that the extent of VI based on the presence of $\mathrm{MCVI}$ and gross VI was independently associated with tumor recurrence and overall survival.

Conclusions: McVI was revealed to be an important risk factor similar to gross VI confined to a segmental/sectional branch in patients with HCC who underwent hepatic resection. This finding should be considered when estimating the stage for prognosis.
\end{abstract}

Keywords: Alpha-fetoprotein, Hepatectomy, Vascular invasion, Tumor node metastasis stage, Tumor recurrence

\section{Background}

Hepatocellular carcinoma (HCC) is one of the most commonly diagnosed cancers and is responsible for a high incidence of cancer-related deaths throughout the world. [1] However, treatment with curative intention, such as hepatic resection, liver transplantation (LT), and locoregional therapies, can only be applied in approximately $30 \%$ of patients with early-stage HCC [2]. Although these therapeutic modalities have improved the overall survival (OS), longterm outcomes remain poor because of high rates of tumor recurrence. Vascular invasion (VI) is a key contributor

\footnotetext{
* Correspondence: dr.wang.hj@gmail.com; wanghj@ajou.ac.kr 'Department of Surgery, School of Medicine, Ajou University, 164 World cup-ro, Yeongtong-gu, Suwon 16499, South Korea

Full list of author information is available at the end of the article
}

to tumor recurrence, which leads to dismal outcomes in patients with $\mathrm{HCC}$ [3].

When HCC tumor progresses, it may invade neighboring vessels [4]. VI by tumor cells is a well-recognized negative prognostic feature of $\mathrm{HCC}$, which has been reflected in official staging systems [5-7]. In the tumor node metastasis (TNM) stage based on the criteria of the Liver Cancer Study Group of Japan (LCSGJ), VI is one of three factors for determining the $\mathrm{T}$ stage with tumor size and numbers [6]. According to the Barcelona Clinic for Liver Cancer (BCLC) staging, HCC with gross VI is classified as advanced stage, which most likely will not benefit from curative treatment [7]. However, it remains unclear how much microvascular invasion (McVI) provides prognostic information for patients with HCC from the viewpoint of the extent of tumor invasion or extension. 
Studies evaluating patients stratified by various predictors of recurrence risk have identified McVI as a factor that can affect the prognosis of postoperative recurrence $[8,9]$. McVI is also reported in several studies to be an important risk factor for HCC recurrence after LT [10, 11]. Subsequent studies have focused on preoperative prediction of McVI to aid the decision-making process for optimal treatment option in patients with $\mathrm{HCC}[12,13]$.

Considered as the first step of metastatic dissemination via the vascular route, prognostic impact of $\mathrm{McVI}$ may be intuitively thought to be placed between non-VI and gross invasion of vessels. However, there is no strong evidence to support this speculation. The protocol developed by the College of American Pathologists considers McVI the same as gross VI confined to segmen$\mathrm{tal} /$ sectional branches of $\mathrm{HCC}$ on the current American Joint Committee on Cancer (AJCC)/International Union for Cancer Control (UICC) tumor TNM staging system [14]. Unfortunately, there is no mention of the prognostic significance of McVI on other staging systems, such as LCSGJ TNM or BCLC $[6,7]$. In this retrospective study, we aimed to clarify the importance of McVI as the degree of local tumor invasion or extension in tumor stage for HCC.

\section{Methods}

A retrospective analysis was performed on a database of patients who underwent surgical procedures for HCC at our center between September 1994 and December 2012. Data were extracted from prospectively collected database records, which included demographics, etiology of underlying liver disease, pathological findings of the specimen, surgical results, and oncological outcomes. Patients lost during follow-up were censored.

We preferentially considered and attempted surgical resection for all patients newly diagnosed with HCC in the Department of Surgery and all referred patients from the Department of Gastroenterology and other institutions if liver function was preserved and the state of HCC was not technically inoperable. Liver function was assessed by the Child-Turcotte-Pugh (CTP) classification and indocyanine green retention rate at $15 \mathrm{~min}$ (ICG-R15) value. For many years, our approach to determine the extent of resection has been based on a prediction scoring system [15]. We did not abandon hepatic resection because of the existence or extent of gross VI. Major hepatic resection was defined as the removal of three or more segments according to the Brisbane classification [16]. Intraoperative ultrasound was routinely used to detect any additional nodules and to aid in the determination of the most optimal resection plane.

Tumors were staged based on postoperative pathological findings according to the AJCC/UICC TNM and
LCSGJ staging system [5, 6]. McVI was defined by a tumor within a vascular space lined by endothelium, identified only on microscopy in the capsule or noncapsular fibrous septa or liver tissue surrounding the tumor [17]. In all cases, tumor grade was defined by the poorest degree of differentiation using the Edmondson-Steiner grades, identified within the tumor upon pathological analysis of the entire specimen [18]. Portal vein tumor thrombus (PVTT) and hepatic vein tumor thrombus (HVTT) was classified into five and four groups, respectively, according to the General Rules for the Study of Primary Liver Cancer by the Korean Liver Cancer Study Group [19].

Follow-up investigations consisted of imaging studies with serum $\alpha$-fetoprotein (AFP) level. Biochemical liver function tests, AFP level, and abdominal computed tomography (CT) scan were conducted every 3 months after discharge during the first 2 years and approximately every 3-6 months for the following years. Tumor recurrence was diagnosed by the combination of elevated tumor markers and consistent radiological findings. If recurrence was highly suspected without clear evidence on an imaging study, hepatic arteriography and lipiodol CT scans were performed. Patients with tumor recurrence were managed with various therapeutic modalities, including local ablation, re-resection, and salvage LT. Patients with multiple or large tumors and/or hepatic dysfunction underwent transcatheter arterial chemoembolization (TACE). Targeted therapy with sorafenib and radiation therapy were also adopted for advanced or metastatic tumors.

\section{Statistical analysis}

Variables preoperatively and pathologically stratified were analyzed using univariate and multivariate analyses to determine independent predictors of oncological outcome. All continuous variables were expressed as mean \pm standard deviation or median (minimum-maximum range). The optimal cutoff values for continuous variables for use in the Kaplan-Meier survival analyses were estimated by receiver operating characteristic (ROC) curve analysis. Survival rates and curves were estimated using the Kaplan-Meier method and compared using the log rank test. Multivariate analysis was performed using the Cox regression proportional hazards model to identify independent factors that determined recurrencefree survival (RFS) and OS. All statistical analyses were performed using $R$-packages, version 3.3.1 [20]. All $P$ values $<0.05$ were considered statistically significant.

\section{Results}

A total of 884 surgical procedures for HCC were performed during the study period. Patients who underwent primary hepatic resection were eligible for the study. 
Exclusion criteria were patients who underwent primary LT for HCC $(n=93)$ and those undergoing reoperation $(n=79)$, such as repeated hepatic resection, salvage or repeated LT, and hepatic resection following LT. The diagnosis of $\mathrm{HCC}$ was confirmed by pathological examination in all cases. A total of 33 patients who had combined HCC and cholangiocarcinoma and three with distant metastasis at the operation time were also excluded. This retrospective study was performed on the remaining 676 patients (Fig. 1), and their clinicopathological details are summarized in Table 1 . There were 530 male $(78.4 \%)$ and 146 female (21.6\%) patients (median age, 52 years; range, $20-76$ years). Among the patients, $516(78.5 \%)$ tested positive for serum hepatitis B surface antigen and $38(6 \%)$ tested positive for hepatitis $\mathrm{C}$ antibody; 36 (5.3\%) and 14 (2.1\%) patients had CTP classes B and $C$, respectively, and 623 (92.6\%) had class A disease. Serum AFP level was normal in 197 patients (29.6\%), abnormal but less than $400 \mathrm{ng} / \mathrm{mL}$ in 251 (37.7\%), and more than $1000 \mathrm{ng} / \mathrm{mL}$ in 217 (32.7\%). Two hundred thirty-two (40.0\%) patients underwent preoperative TACE. Among the operations, 262 (38.7\%; approximately $4 / 10$ rate) were major hepatic resections, whereas $184(27.2 \%)$ were segmentectomies or bisegmentectomies and 230 (34.1\%) were minor resections.

Overall median follow-up period was 40 (1-204) months. The 90-day mortality rate after hepatic resection because of post-hepatectomy liver failure or sepsis was $1.9 \%$ (13 of 676). During the follow-up period, $55.1 \%$ (365 of 663) of the patients had tumor recurrence and $35.6 \%$ (236 of 663 ) died. The 5-year OS and RFS rates were 63.3 and $42.6 \%$, respectively.

Pathological analysis postoperatively revealed that 328 patients (48.5\%) had combined McVI: 193 had McVI without and 135 had McVI with gross VI. According to the extent of PVTT, 537 patients (79.4\%) had Vp0, 58 (8.6\%) had Vp1, 41 (6.1\%) had Vp2, 22 (3.3\%) had Vp3, and $18(2.7 \%)$ had Vp4. A total of 29 patients $(4.3 \%)$ had tumor with hepatic vein invasion. Based on the extent of HVTT, the patients were classified into four groups: $\mathrm{Vv0}$ $(n=647), \mathrm{Vv1}(n=19), \mathrm{Vv} 2(n=5)$, and Vv3 $(n=5)$. The patients were also divided into four groups based on the existence of McVI and extent of gross VI: group A, no McVI or gross VI; group B, McVI without gross VI; group C, VI confined to segmental/sectional branches (Vp1-2 or Vv1); and group D, gross VI within/beyond major vascular branches (Vp3-4 or $\mathrm{Vv} 2-3)$. The relationship between groups $\mathrm{A}-\mathrm{D}$ and the clinicopathological factors are shown in Table 1.

We observed marked differences in groups of patients according to preoperative platelet count, aspartate aminotransferase (AST) and AFP level, and tumor size. There were also significant differences in the percentage of major operations, multiplicity of tumors, intrahepatic metastasis, tumor histology, histological involvement of the resection margin, and tumor stage. Group D had the highest preoperative platelet counts, AST and AFP levels, and tumor sizes. In comparison, group A had the lowest values of these parameters. In addition, group $\mathrm{D}$ had the highest rate of major hepatic resection (91.1\%), followed by group C (55.4\%), whereas group A had the lowest rate of major hepatic resection (26.0\%). We observed that $31.1 \%$ of patients in group D had multiple tumors, whereas $85.7 \%$ in group A had a single tumor. Moreover, group D had the highest proportions of intrahepatic metastasis and worse tumor histological grade (66.7 and $58.1 \%$, respectively), followed by group C (53.4 and 54.0\%, respectively). In addition, group D had the highest rate of positive surgical margins, followed by group C (36.4 and 22.5\%, respectively), whereas group A had the lowest rate of intrahepatic metastasis (20.9\%), worse tumor histological grade (21.7\%), and positive surgical margins (6.3\%). When comparing the survival curves according to these four

\section{Operated hepatocellular carcinomas $(\mathrm{HCC})(\mathbf{n}=\mathbf{8 8 4})$}

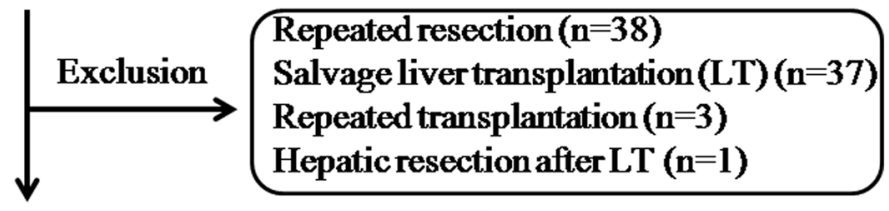

Primary surgical treatment $(\mathbf{n}=\mathbf{8 0 5})$

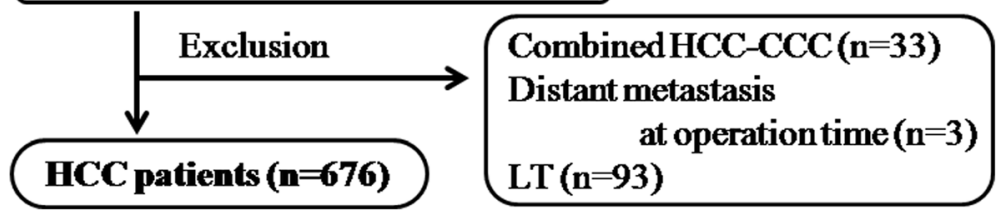

Fig. 1 Flow diagram shows the selection of patients who were eligible for this study. CCC cholangiocarcinoma 
Table 1 Comparison of clinicopathological data for patients classified into four groups

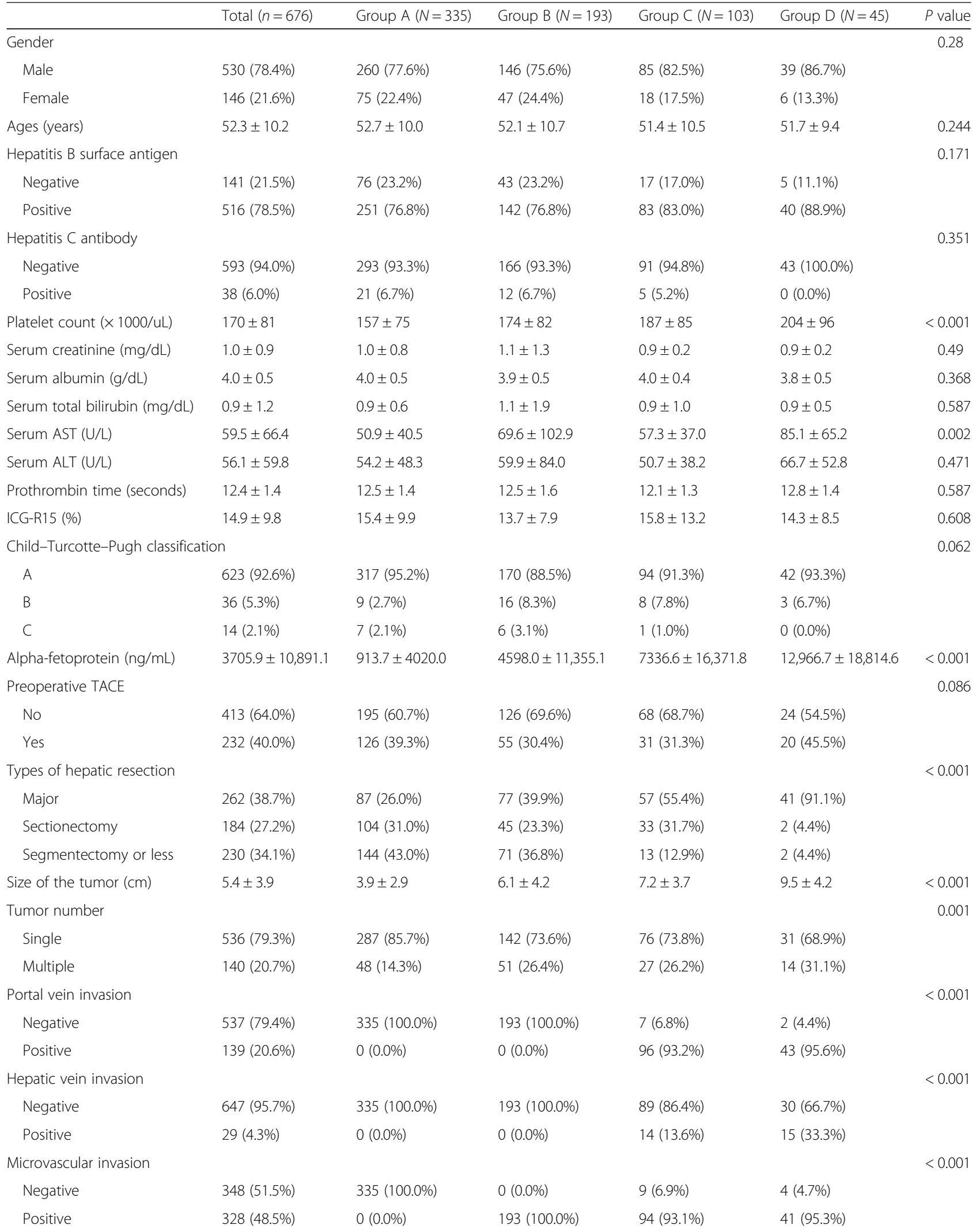


Table 1 Comparison of clinicopathological data for patients classified into four groups (Continued)

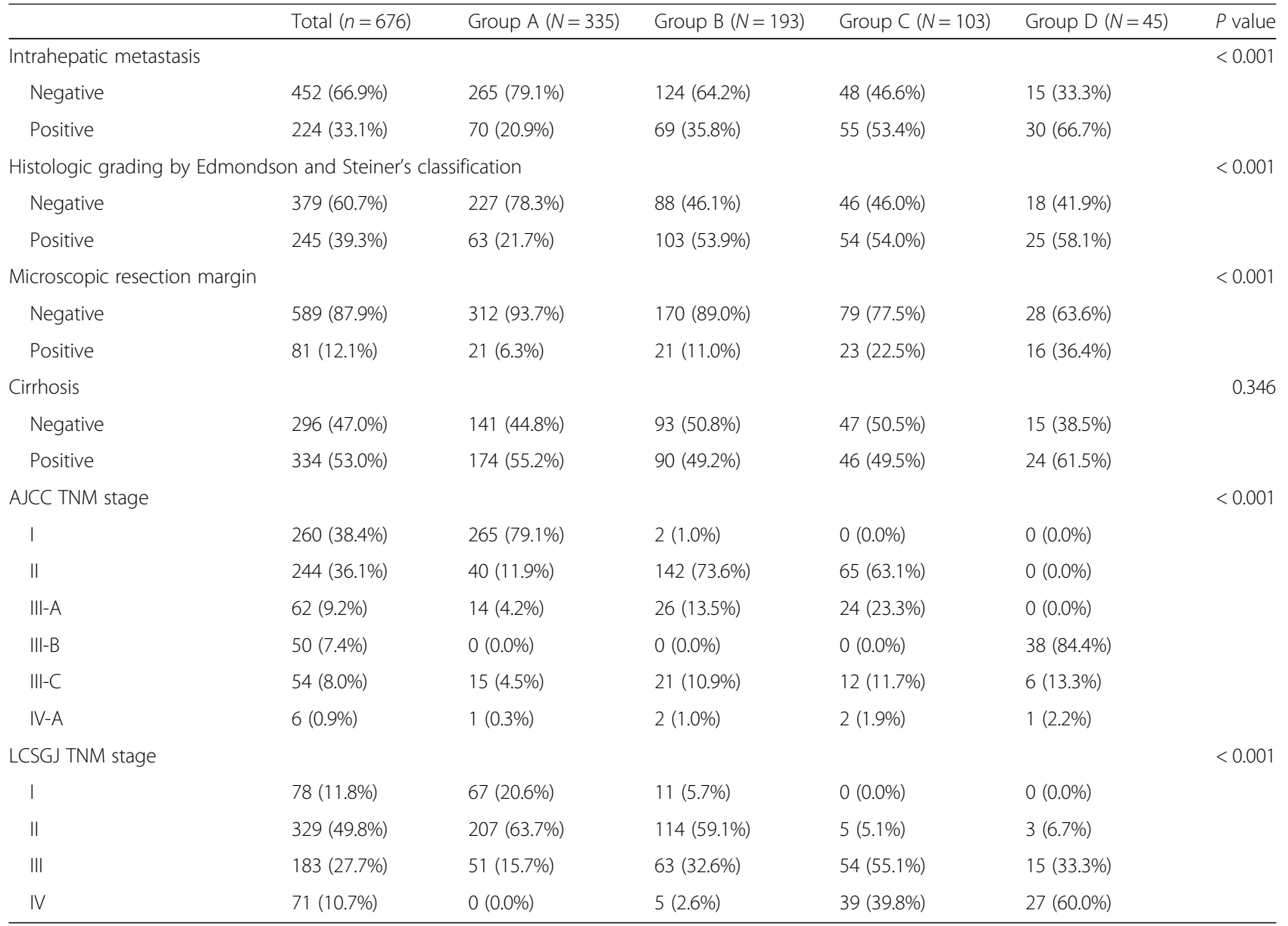

Group A no microvascular invasion (McVI) or gross vascular invasion (VI), Group B McVI without gross VI, Group C VI confined to segmental/sectional branches, Group D gross VI within/beyond major vascular branches, ICG-R15 indocyanine green retention rate at $15 \mathrm{~min}$, TACE transcatheter arterial chemoembolization, AJCC TNM American Joint Committee on Cancer Tumor Node Metastasis, LCSGJ the Liver Cancer Study Group of Japan

groups, group D demonstrated significantly worse survival compared to the other groups: RFS and OS for groups A versus $\mathrm{D}(P<0.001)$, B versus $\mathrm{D}(P<0.001)$, and $\mathrm{C}$ versus $\mathrm{D}(P=0.001)$. Moreover, groups $\mathrm{B}$ and $\mathrm{C}$ showed markedly worse outcomes than group A: RFS and $O S$ for groups $A$ versus $B(P<0.001)$ and $A$ versus $\mathrm{C}(P<0.001)$. However, no significant differences in RFS and OS were noted between groups $B$ and C: $5-$ year RFS rates, 29.8 and $27.7 \%$, respectively $(P=0.18)$; 5 -year OS rates, 56.4 and $56.5 \%$, respectively $(P=0.43$; Fig. 2). Therefore, patients were reclassified into three groups (groups $\mathrm{A}$ vs. $\mathrm{B} / \mathrm{C}$ vs. D) for further analysis in a multivariate model.

Univariate analysis according to clinicopathological factors was used to find predictors of tumor recurrence and survival. Cutoff values for the continuous variables (preoperative platelet count, AST and AFP levels, etc.) were calculated by ROC curve analysis (Table 2). Multivariate analysis revealed predictors that were independently associated with tumor recurrence and OS. The extent of
VI (groups A vs. B/C vs. D), higher AST level, existence of intrahepatic metastasis, larger tumor size, elevated ICGR15 value, prolonged prothrombin time, liver cirrhosis, and advanced tumor stage were independent risk factors for tumor recurrence. Albumin level over $4 \mathrm{~g} / \mathrm{dL}$ was a positive risk factor for prognosis (Fig. 3a). Among the abovementioned risk factors, larger tumor size and elevated ICG-R15 value were not significantly related to poor OS. Worse histological grade and positive surgical margins were independent predictive factors of worse survival (Fig. 3b).

\section{Discussion}

Our study demonstrated the clinical significance of $\mathrm{McVI}$ in a manner that has not been used in previous similar studies. When the influence of McVI was analyzed, tumor recurrence and survival rates of patients with $\mathrm{HCC}$ and McVI without gross VI (group B) were not different from those of patients with gross VI confined to segmental/sectional braches (group C) after 

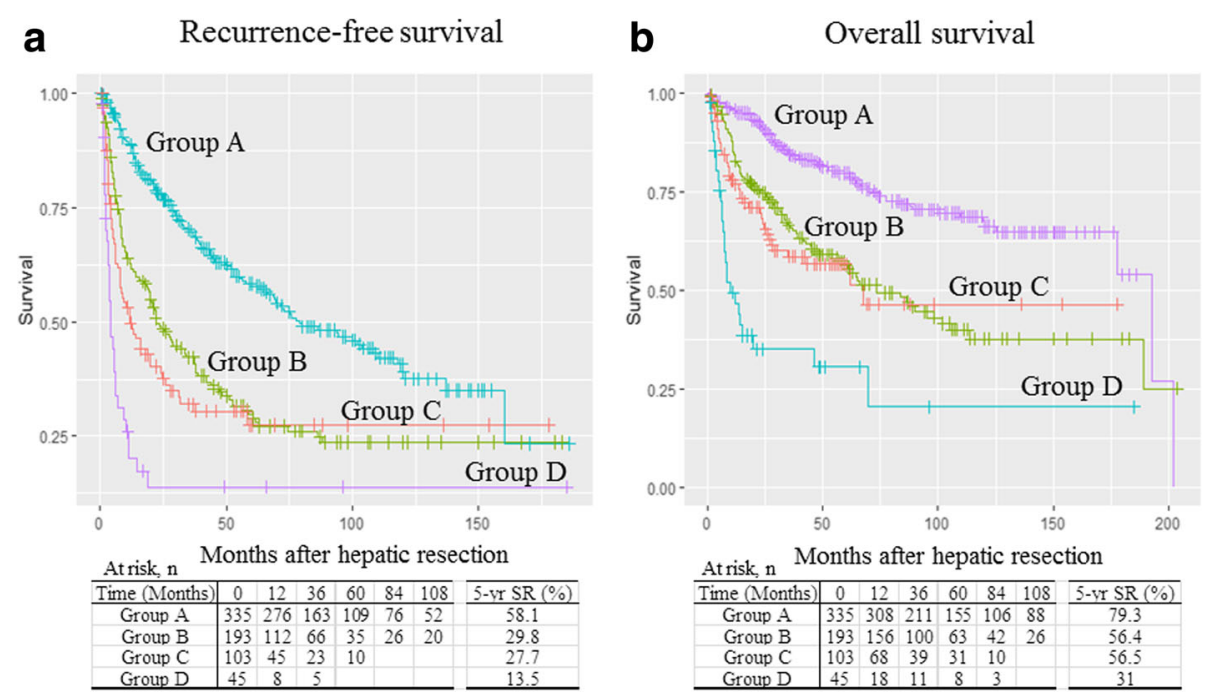

Fig. 2 Comparison of a recurrence-free and $\mathbf{b}$ overall survival of patients stratified into groups A-D. No significant changes are seen between groups $B$ and $C$ (recurrence-free survival, $P=0.18$; overall survival, $P=0.43$ ). SR survival rate

hepatic resection. To the best of our knowledge, no study has directly compared the relative importance of McVI and gross VI on tumor recurrence and long-term survival of patients with HCC undergoing hepatic resection.

Previous studies have shown that McVI is an important factor affecting the prognosis of patients with HCC, especially after hepatic resection or LT [8-11]. However, it is difficult to find studies dealing with the significance of McVI as the degree of local tumor invasion or extension, despite the instinctive guess that it might be an intermediate state of VI between nonvascular invasion and gross tumor invasion of neighboring segmental vessels. We generally assumed that the risk of tumor recurrence, as well as death, would be significantly lower in patients with HCC and McVI without gross VI (group B) than in those with gross VI confined to the segmental/sectional branch (group C). Contrary to our expectation, McVI has similar prognostic power compared with gross VI confined to the segmental/sectional branch (Fig. 2).

Despite its importance, official staging systems, such as the LCSGJ TNM, and BCLC staging systems, contain no mention of McVI $[6,7]$. The protocol developed by the College of American Pathologists considers McVI the same as gross VI of HCC on the AJCC/UICC TNM staging system, although related studies are difficult to find [14]. Then, we focused on whether tumor stage would be influenced by McVI. Our primary goal for this study has been to evaluate the importance of McVI in tumor stage for HCC. In the present study, patients with HCC and McVI without gross VI (group B) had similar outcomes of tumor recurrence and survival compared with those with gross VI confined to segmental/sectional branches (group C). When compared with patients with gross VI within/beyond major vascular branches (group D), patients in group $\mathrm{B} / \mathrm{C}$ had lower rates of tumor recurrence and good survival (Fig. 3). Our results tended to support the protocol of the College of American Pathologists.

While survival outcomes are notoriously worse in patients with gross VI than in those without gross VI (group A), those with HCC with gross VI confined to segmental/sectional branches (group C) had better outcomes than those with gross VI within/beyond major vascular branches (group D). Several studies have dealt with the extent of gross VI and its clinical impact on HCC [21, 22]. Survival outcomes of these previous studies are comparable to those of our study. The essential of cancer surgery is complete removal of tumor with free and safe margins. From the viewpoint of surgical principle, resection of a tumor with gross VI isolated within segmental/sectional branches could be considered as curative intention treatment through major hepatic resection without exposure of tumor thrombus margins on the portal or hepatic vein. However, resection of tumor with gross VI within/beyond major vascular branches should be considered as palliative treatment because exposure of tumor thrombus inside the vessels is not avoidable.

In the BCLC staging system, patients with $\mathrm{HCC}$ and VI (preoperative gross VI on image studies) are classified as having stage $\mathrm{C}$ disease and guided into treatments with palliative intent [7]. There is no mention of McVI because the BCLC system is designed to guide treatment according to preoperative patient information and McVI can be postoperatively confirmed through resected specimen. Therefore, there have been efforts to preoperatively detect McVI in HCC. Tools, such as performance 
Table 2 Univariate analysis of factors predictive of recurrence-free and overall survival

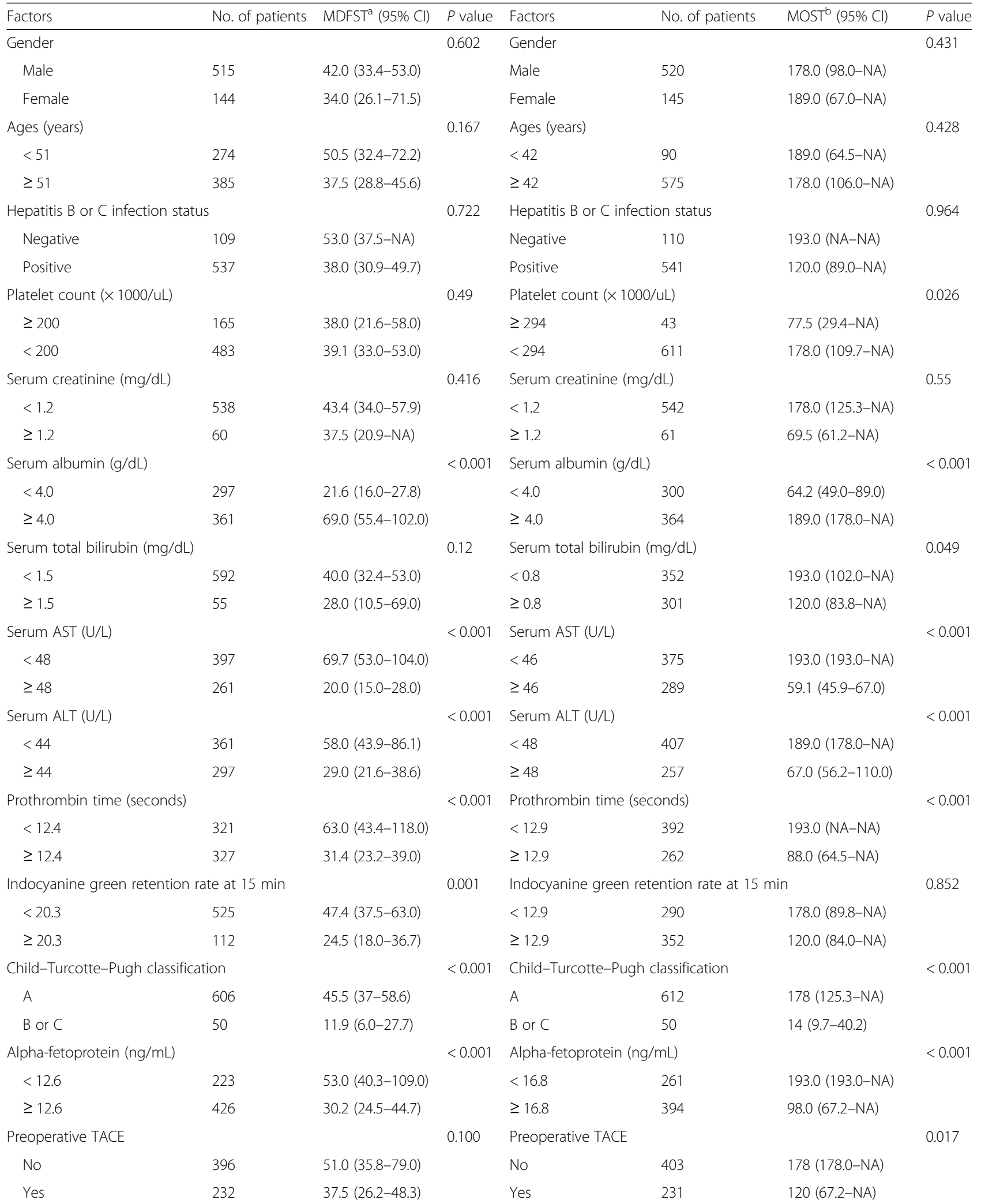


Table 2 Univariate analysis of factors predictive of recurrence-free and overall survival (Continued)

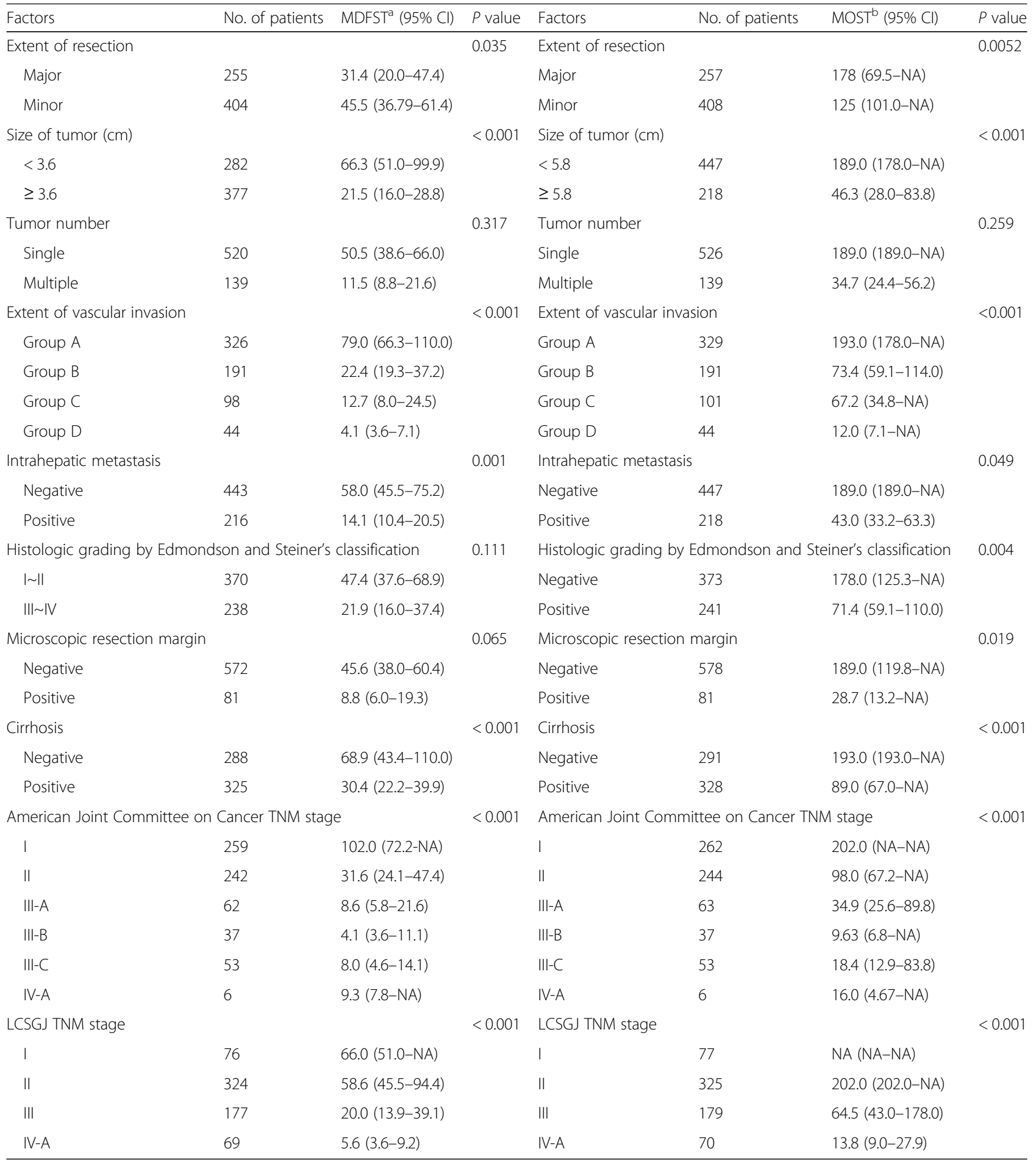

CI confidence interval, NA not available, TNM tumor node metastasis, LCSGJ the Liver Cancer Study Group of Japan ${ }^{a}$ Median disease-free survival time (month)

${ }^{\mathrm{b}}$ Median overall survival time (month)

of prothrombin induced by vitamin $\mathrm{K}$ absence-II and fluorodeoxyglucose-positron emission tomography, have already been suggested to preoperatively predict $\mathrm{McVI}$ of HCC [23, 24]. Moreover, several studies have been conducted using radiological imaging, molecules or gene expression from tumor, and other preoperative 

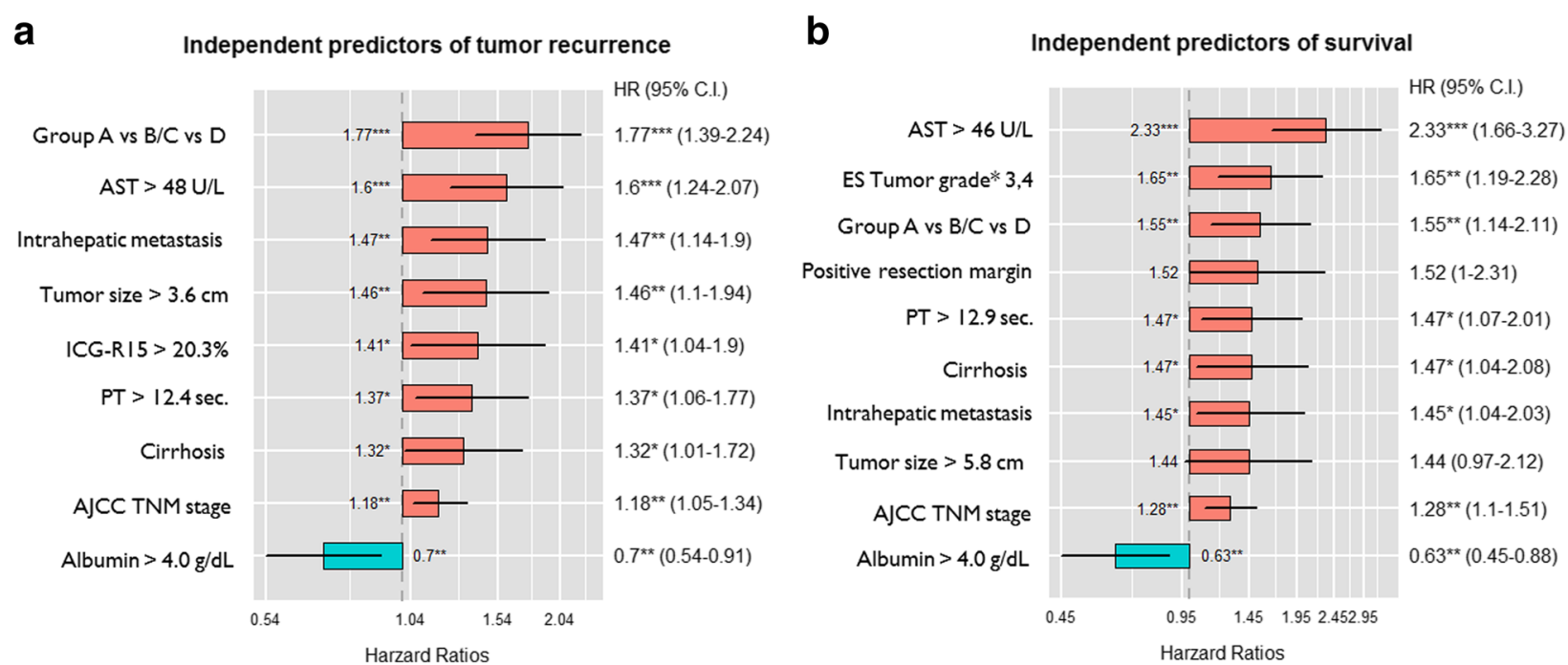

Fig. 3 Summary of statistically significant clinicopathological factors on a recurrence-free survival and $\mathbf{b}$ overall survival using the Cox regression proportional hazards model. AJCC TNM American Joint Committee on Cancer Tumor Node Metastasis, AST aspartate aminotransferase, ES Edmondson-Steiner classification, HR hazard ratio, C.I. confidence interval, ICG-R15 indocyanine green retention rate at 15 min, PT prothrombin time

tumor characteristics [25-27]. At this point, a practical question can be raised. Should patients with $\mathrm{HCC}$ be guided to palliative treatment if McVI can be preoperatively determined? We suggest that treatments with curative intent should be recommended for patients with $\mathrm{HCC}$ if they have good liver function, based on the results of our current study.

VI of HCC tumor is considered to be a reflection of aggressiveness and has a well-known negative prognostic impact after hepatic resection [28]. However, little information is available regarding this tumor progression mechanism, which remains to be elucidated. A possible postulation is that portal vein or hepatic vein tumor invasion may simply be an effect of tumor topography, which means that this aggressive phenomenon may happen only because of the close anatomical proximity to neighboring vessels. A study comparing gene expressions between primary tumors and their paired portal vein tumor thrombi has demonstrated only a small difference [29]. However, studies focused on the mechanism of tumor metastasis have demonstrated the importance of phenotype changes in individual tumor cells [30, 31]. Recently, genomic studies have shown that unique genes and noncoding RNAs may have an important role in this mechanism [32, 33].

Serum levels of aspartate aminotransferase (AST) are one of the important prognostic factors after hepatic resection for HCC in this study. A study demonstrated that higher AST levels are positively correlated with an influx of hepatitis B virus [34]. In this study, $78.5 \%$ of patients have chronic B-viral hepatitis. Advancing underlying liver diseases may also be related to mitochondrial injury, which leads the release of AST [35]. So, elevated AST level may be indirectly reflecting the progress of hepatitis B. Multiple studies have supported that sustained viremia has a role in recurrence of hepatitis B virus-related HCC, and prevention effect of anti-viral therapy for recurrence [36].

The present study limitations include its retrospective nature and nonrandomized design, even though the data were prospectively collected. Furthermore, there was little information on important patient perioperative status, such as antiviral drug use, postoperative progression of underlying liver disease, or exposure to other carcinogens including alcohols, which have been considered to influence tumor recurrence or de novo malignancy. External validation of meaningful findings in this study is also needed in a multicenter-organized database setting. Unfortunately, there is a lack of clarity in the definition of McVI, leading to inter- and intra-pathologist variability in the evaluation of McVI in HCC [17]. However, all tumor tissues were evaluated by one liver-specialized pathologist with over 25 years of experience in this study. There is an attempt to establish a definition of McVI in HCC, using general histopathological principles, requiring prospective validation [37].

\section{Conclusion}

McVI showed similar clinical significance compared with gross VI confined to segmental/sectional branches as a risk factor for tumor recurrence and poor survival of patients with HCC. Therefore, this study recommends considering McVI when estimating the tumor stage to predict the prognosis and to plan follow-up surveillance and additional treatment for patients with HCC. 


\section{Abbreviations}

AFP: a-fetoprotein; AJCC: American Joint Committee on Cancer; AST: Aspartate aminotransferase; BCLC: Barcelona Clinic for Liver Cancer; Cl: Confidence interval; CT: Computed tomography; CTP: Child-TurcottePugh; HCC: Hepatocellular carcinoma; HR: Hazard ratio; HVTT: Hepatic vein tumor thrombus; ICG-R15: Indocyanine green retention rate at $15 \mathrm{~min}$; LCSGJ: Liver Cancer Study Group of Japan; LT: Liver transplantation; McVI: Microvascular invasion; OS: Overall survival; PVTT: Portal vein tumor thrombus; RFS: Recurrence-free survival; ROC: Receiver operating characteristic; TNM: Tumor node metastasis; UICC: International Union for Cancer Control; VI: Vascular invasion

\section{Acknowledgements}

Not applicable.

\section{Funding}

This work was supported by the National Research Foundation of Korea (NRF) grant funded by the Korea government (MSIP; Ministry of Science, IC \& Future Planning) (No. 2017R1C1B1008436 and No. 2017R1C1B5076680).

\section{Availability of data and materials}

Data sharing is not applicable to this article as no datasets were generated or analyzed during the current study.

\section{Authors' contributions}

YKP, SKS, and HJW designed the study. YKP, SKS, BWK, SKP, and HJW analyzed and interpreted the data. YKP, SKS, and HJW wrote and revised the manuscript. BWK, SKP, and CWC reviewed the manuscript. All authors discussed the study and commented on the manuscript. All authors read and approved the final manuscript.

\section{Ethics approval and consent to participate}

This study was approved by the Institutional Review Board of Ajou University Medical Center (Suwon, South Korea), with the need for informed consent waived.

\section{Consent for publication}

Not applicable.

\section{Competing interests}

The authors declare that they have no competing interests.

\section{Publisher's Note}

Springer Nature remains neutral with regard to jurisdictional claims in published maps and institutional affiliations.

\section{Author details}

'Department of Surgery, School of Medicine, Ajou University, 164 World cup-ro, Yeongtong-gu, Suwon 16499, South Korea. ${ }^{2}$ Department of Surgery, International St. Mary's Hospital, Catholic Kwandong University, Incheon, South Korea. ${ }^{3}$ Department of Supercomputing, M\&S Technology Development, Korea Institute of Science and Technology Information, Daejeon, South Korea.

\section{Received: 3 September 2017 Accepted: 5 December 2017} Published online: 19 December 2017

\section{References}

1. Torre LA, Bray F, Siegel RL, Ferlay J, Lortet-Tieulent J, Jemal A. Global cancer statistics. CA Cancer J Clin. 2015;65:87-108.

2. Llovet JM. Updated treatment approach to hepatocellular carcinoma. J Gastroenterol. 2005;40:225-35

3. Thuluvath PJ. Vascular invasion is the most important predictor of survival in HCC, but how do we find it? J Clin Gastroenterol. 2009;43:101-2.

4. Liu CC, Chang TC, Lin YT, YL Y, Ko BS, Sung LY, Liou JY. Paracrine regulation of matrix metalloproteinase contributes to cancer cell invasion by hepatocellular carcinoma-secreted 14-3-3б. Oncotarget. 2016;7:36988-99.

5. Edge SE, Byrd DR, Campton CC, Fritz A, Greene F, Trotti A. AJCC cancer staging manual. 7th ed. New York: Springer; 2009.

6. Liver Cancer Study Group of Japan. General rules for the clinical and pathological study of primary liver cancer, $2^{\text {nd }}$ English ed. Tokyo: Kanehara; 2003.
7. Llovet JM, Bru C, Bruix J. Prognosis of hepatocellular carcinoma: the BCLC staging classification. Semin Liver Dis. 1999;19:329-38.

8. Lim KC, Chow PK, Allen JC, et al. Microvascular invasion is a better predictor of tumor recurrence and overall survival following surgical resection for hepatocellular carcinoma compared to the Milan criteria. Ann Surg. 2011; 254:108-13.

9. Fuks D, Dokmak S, Paradis V, Diouf M, Durand F, Belghiti J. Benefit of initial resection of hepatocellular carcinoma followed by transplantation in case of recurrence: an intention-to-treat analysis. Hepatology. 2012;55:132-40.

10. Iguchi T, Shirabe K, Aishima S, Wang H, Fujita N, Ninomiya M, Yamashita Y, Ikegami T, Uchiyama H, Yoshizumi T, Oda Y, Maehara Y. New pathologic stratification of microvascular invasion in hepatocellular carcinoma: predicting prognosis after living-donor liver transplantation. Transplantation. 2015;99: 1236-42.

11. Donat M, Alonso S, Pereira F, Ferrero E, Carrión L, Acin-Gándara D, Moreno E. Impact of histological factors of hepatocellular carcinoma on the outcome of liver transplantation. Transplant Proc. 2016:48:1968-77.

12. Renzulli M, Brocchi S, Cucchetti A, Mazzotti F, Mosconi C, Sportoletti C, Brandi G, Pinna AD, Golfieri R. Can current preoperative imaging be used to detect microvascular invasion of hepatocellular carcinoma? Radiology. 2016; 279:432-42.

13. Schlichtemeier SM, Pang TC, Williams NE, Gill AJ, Smith RC, Samra JS, Lam W, Hollands M, Richardson AJ, Pleass HC, Nozawa S, Albania M, Hugh TJ. A pre-operative clinical model to predict microvascular invasion and longterm outcome after resection of hepatocellular cancer: the Australian experience. Eur J Surg Oncol. 2016;42:1576-83.

14. Kakar S, Shi C, Fitzgibbons PL, Frankel WL, Krasinskas AM, Mino-Kenudson M Pawlik T, Vauthey JN, Washington MK. Protocol for the examination of specimens from patients with hepatocellular carcinoma. Posted June 2017, Version Hepatocellular 4.0.0.0. Available at: http://www.cap.org.

15. Yamanaka N, Okamoto E, Oriyama T, Fujimoto J, Furukawa K, Kawamura E, Tanaka T, Tomoda F. A prediction scoring system to select the surgical treatment of liver cancer. Further refinement based on 10 years of use. Ann Surg. 1994;219:342-6.

16. Strasberg SM. Nomenclature of hepatic anatomy and resections: a review of the Brisbane 2000 system. J Hepato-Biliary-Pancreat Surg. 2005;12:351-5.

17. Fan L, Mac MT, Frishberg DP, Fan X, Dhall D, Balzer BL, Geller SA, Wang HL. Interobserver and intraobserver variability in evaluating vascular invasion in hepatocellular carcinoma. J Gastroenterol Hepatol. 2010;25(9):1556-61.

18. Edmonson HA, Steiner PE. Primary carcinoma of the liver. Cancer. 1954;7: 462-503.

19. Korean Liver Cancer Study Group and National Cancer Center Korea. Practice guidelines for management of hepatocellular carcinoma 2009. Korean J Hepatol. 2009;15:391-423.

20. R Core Team. R: a language and environment for statistical computing. Vienna: R Foundation for Statistical Computing; 2016. URL https://www. R-project.org/

21. Shi J, Lai EC, Li N, Guo WX, Xue J, Lau WY, MC W, Cheng SQ. Surgical treatment of hepatocellular carcinoma with portal vein tumor thrombus. Ann Surg Oncol. 2010;17:2073-80.

22. Peng ZW, Guo RP, Zhang YJ, Lin XJ, Chen MS, Lau WY. Hepatic resection versus transcatheter arterial chemoembolization for the treatment of hepatocellular carcinoma with portal vein tumor thrombus. Cancer. 2012; 118:4725-36.

23. Shirabe K, Toshima T, Kimura K, Yamashita Y, Ikeda T, Ikegami T, Yoshizumi $T$, Abe K, Aishima S, Maehara Y. New scoring system for prediction of microvascular invasion in patients with hepatocellular carcinoma. Liver Int. 2014;34:937-41.

24. Kobayashi T, Aikata H, Honda F, Nakano N, Nakamura Y, Hatooka M, Morio K, Morio R, Fukuhara T, Masaki K, Nagaoki Y, Kawaoka T, Tsuge M, Hiramatsu A, Imamura M, Kawakami Y, Ohdan H, Awai K, Chayama K. Preoperative fluorine 18 fluorodeoxyglucose positron emission tomography/computed tomography for prediction of microvascular invasion in small hepatocellular carcinoma. J Comput Assist Tomogr. 2016:40:524-30.

25. Jhaveri KS, Cleary SP, Fischer S, Haider MA, Pargoankar V, Khalidi K, Moshonov $\mathrm{H}$, Gallinger S. Blood oxygen level-dependent liver MRI: can it predict microvascular invasion in HCC? J Magn Reson Imaging. 2013;37:692-9.

26. Poté N, Alexandrov T, Le Faouder J, Laouirem S, Léger T, Mebarki M, Belghit J, Camadro JM, Bedossa P, Paradis V. Imaging mass spectrometry reveals modified forms of histone $\mathrm{H} 4$ as new biomarkers of microvascular invasion in hepatocellular carcinomas. Hepatology. 2013;58:983-94. 
27. Eguchi S, Takatsuki M, Hidaka M, Soyama A, Tomonaga T, Muraoka I, Kanematsu T. Predictor for histological microvascular invasion of hepatocellular carcinoma: a lesson from 229 consecutive cases of curative liver resection. World J Surg. 2010;34:1034-8.

28. Liu W, Wang K, Bao Q, Sun Y, Xing BC, Hao S, Fan P, Chen S, Tu C, Wan C. Hepatic resection provided long-term survival for patients with intermediate and advanced-stage resectable hepatocellular carcinoma. World I Surg Oncol. 2016;14:62.

29. Ye Q, Qin LX, Forgues $M$, He P, Kim JW, Peng AC, Simon R, Li Y, Robles Al, Chen Y, Ma ZC, ZQ W, Ye SL, Liu YK, Tang ZY, Wang XW. Predicting hepatitis B virus-positive metastatic hepatocellular carcinomas using gene expression profiling and supervised machine learning. Nat Med. 2003;9:416-23.

30. Guo HB, Zhang Y, Chen HL. Relationship between metastasis-associated phenotypes and $\mathrm{N}$-glycan structure of surface glycoproteins in human hepatocarcinoma cells. J Cancer Res Clin Oncol. 2001;127(4):231-6.

31. Bacigalupo ML, Manzi M, Espelt MV, Gentilini LD, Compagno D, Laderach DJ, Wolfenstein-Todel C, Rabinovich GA, Troncoso MF. Galectin-1 triggers epithelial-mesenchymal transition in human hepatocellular carcinoma cells. J Cell Physiol. 2015;230:1298-309.

32. Choi JH, Kim MJ, Park YK, Im JY, Kwon SM, Kim HC, Woo HG, Wang HJ. Mutations acquired by hepatocellular carcinoma recurrence give rise to an aggressive phenotype. Oncotarget. 2017;8:22903-16.

33. Budhu A, Jia HL, Forgues M, Liu CG, Goldstein D, Lam A, Zanetti KA, Ye QH, Qin LX, Croce CM, Tang ZY, Wang XW. Identification of metastasis-related microRNAs in hepatocellular carcinoma. Hepatology. 2008;47:897-907.

34. Witjes $C D$, IJzermans JN, van der Eijk AA, Hansen BE, Verhoef $C$, de Man RA. Quantitative HBV DNA and AST are strong predictors for survival after HCC detection in chronic HBV patients. Neth J Med. 2011;69:508-13.

35. Rej R. Aminotransferases in disease. Clin Lab Med. 1989;9:667-87.

36. Ishikawa T. Anti-viral therapy to reduce recurrence and improve survival in hepatitis B virus-related hepatocellular carcinoma. World J Gastroenterol. 2013;19:8861-6.

37. Rodríguez-Perálvarez M, Luong TV, Andreana L, Meyer T, Dhillon AP, Burroughs AK. A systematic review of microvascular invasion in hepatocellular carcinoma: diagnostic and prognostic variability. Ann Surg Oncol. 2013;20:325-39.

\section{Submit your next manuscript to BioMed Central and we will help you at every step:}

- We accept pre-submission inquiries

- Our selector tool helps you to find the most relevant journal

- We provide round the clock customer support

- Convenient online submission

- Thorough peer review

- Inclusion in PubMed and all major indexing services

- Maximum visibility for your research

Submit your manuscript at www.biomedcentral.com/submit 